\title{
EFFECT OF THE CHARGE STATE ON THE PHOTOLUMINESCENCE SPECTRA OF MELANIN
}

\author{
A.O. Kostetskyi ${ }^{a}$, Yu.P. Piryatinski ${ }^{a}$, A.B. Verbitsky ${ }^{a}$, P.M. Lutsyk ${ }^{b, a}$, \\ A.G. Rozhin ${ }^{b}$ \\ a Institute of Physics, National Academy of Sciences of Ukraine, \\ 46, Nauky prospekt, 03680 Kyiv, Ukraine \\ ${ }^{b}$ School of Engineering \& Applied Science, Aston University, Aston Triangle, \\ B4 7ET Birmingham, UK
}

\begin{abstract}
Steady-state and time-resolved photoluminescence (PL) spectra of natural and synthetic melanin, as well as melanin composites with surfactant, sodium dodecylbenzenesulfonate, and polymethine dye, astraphloxin, have been studied. The melanin molecules having differently charged peripheral groups with terminal $\mathrm{O}$ atoms and $\mathrm{OH}$ groups mixed with the negatively charged surfactant or positively charged astraphloxin dye feature new PL spectral bands. The kinetics of PL and time-resolved emission spectra for the melanin and the composites were also investigated and discussed in terms of intermolecular complexation, nanoaggregation and formation of charge-transfer states. The studies are aiming to understand the electronic properties of various melanins, particularly natural ones.
\end{abstract}




\section{INTRODUCTION}

The structure and properties of melanins differ depending on their origin, e.g. obtained from natural sources or via synthetic routes. Both natural and synthetic melanins have already found their practical applications [1-6]. The most studied synthetic melanin, eumelanin, has well-defined molecular structure of monomers, such as 5,6-indolequinone, 5,6-dihydroxyindole (DHI) and 5,6-dihydroxyindole carboxylic acid (DHICA). These monomers oxidized to more reactive indolequinones combine into short heterogeneous oligomers [2]. Furthermore, the oligomers aggregate forming nano-sized structures of eumelanin. The 5,6indolequinone monomers are chemically bound together and form flat twodimensional nanoclusters, resembling molecular disks, in which chromophores with different lengths of $\pi-\pi$ conjugation can be distinguished $[4,5]$. The number of molecular disks in the nanoclusters ranges from 4 to 5 [2]. The two-dimensional nanoclusters can subsequently form one-dimensional $\pi$-stacked nanoaggregates with distance between the molecular disks in the stacking about $0.35 \mathrm{~nm}$ [2].

The chemical structure of natural melanins has not yet been finally determined, due to their extremely complex structure. For example, the watersoluble melanin obtained from plant material by extraction and studied previously in our group [4] is close to synthetic melanin based on the 5,6-indolequinone monomers [2]. The structure was established by chromatography and elemental 
analysis, and later confirmed by spectroscopic studies [4-7]. However, it is far from comprehensive understanding of natural melanins properties.

In general, natural and synthetic melanins combine structural properties of organic biopolymers and spectral features of disordered inorganic semiconductors [4]. Essentially, all melanins have a few common structural characteristics that make them similar to organic amorphous semiconductors by their electronic properties. First of all, it is a periodic stack ( $\pi$-stacking) or polymer structure [2] having extended $\pi$-conjugation. Such $\pi$-stacked structure provides a significant anisotropy to nanoclusters of melanin, similar to graphite nanoclusters. Depending on the distance between the molecular nanoclusters, an interaction between the nanoclusters in melanin stacks would change affecting the properties of the material.

Since melanin consists of indole derivatives having positive and negatively charged groups, including terminal reactive groups of $\mathrm{C}=\mathrm{O}, \mathrm{OH}$, and $\mathrm{COOH}$, this advanced material can in principle form complexes of various types acting as an electron acceptor and/or an electron donor. Substances with anionic and cationic properties bind to melanin through ionic interaction complemented by the Van der Waals interaction [8]. A large number of chemicals, such as metals (metal ions), polycyclic aromatic hydrocarbons, organic amines, etc. bind to melanin and are kept in the bound state for a long time. Assumingly, melanin protects surrounding tissues, adsorbing and retaining bound to potentially harmful substances, which are then slowly released at low non-toxic concentrations. 
This way, finding agents able to interact with melanins is crucial in better understanding electronic properties of various melanins. The steady-state and timeresolved photoluminescence (PL) studies of such interaction provide an opportunity to investigate monomeric, excimeric, charge-transfer, and aggregate nature of melanin nanoclusters, as well as might strengthen potential for their practical application in the future.

\section{EXPERIMENTAL}

The steady-state PL spectra were measured using both a USB2000+UV-VIS-ES spectrometer through an optical fiber with a diameter of 600 microns (Ocean Optics B.V.) and by a LifeSpec II spectrofluorometer (Edinburgh Instruments Ltd). To excite the steady-state PL, LLS-270 and LLS-385 light-emitting diodes (Ocean Optics B.V.) were used having maximum of excitation wavelengths at $\lambda_{\mathrm{e}}=270$ and $385 \mathrm{~nm}$, respectively. Time-resolved PL spectra, kinetics of PL, and time-resolved emission spectra (TRES) were studied using the LifeSpec II spectrofluorometer. To excite time-resolved PL in visible range, we used an EPL-405 picosecond pulsed diode laser (Edinburgh Instruments Ltd) with $\lambda_{\mathrm{e}}=(405 \pm 10) \mathrm{nm}$, a power of $5 \mathrm{~mW}$, and a pulse duration of $52 \mathrm{ps}$. To excite the PL in the UV range, we employed EPLED-255 light-emitting diode (Edinburgh Instruments Ltd) with $\lambda_{\mathrm{e}}=$ $(255 \pm 10) \mathrm{nm}$, pulse width $900 \mathrm{ps}$, and a power of $40 \mu \mathrm{W}$.

To determine the lifetime $\tau$ of excited states of molecules, the technique of time-correlated single photon counting (TCSPC) with picosecond temporal 
resolution is used in the LifeSpec II settings. The measured PL intensity $F(t)$ is related to the true decay curve $I(t)$ by the integral equation:

$$
F(t)=\int_{0}^{t} I\left(t-t^{\prime}\right) G\left(t^{\prime}\right) d t^{\prime}
$$

where $G\left(t^{\prime}\right)$ is the instrumental function of the spectrofluorometer, which is the convolution of the Instrument Response Functions (IRF) describing a shape of the excitation pulse.

The resulting time dependence of PL can be approximated by the expression [9]:

$$
I(t)=\sum_{i=1}^{n} A_{i} \exp \left(-\frac{t}{\tau_{i}}\right)
$$

where $\mathrm{i}$ - the serial number, $\tau$ - the lifetime of the excited state, $A_{i}$ - the weighting coefficient.

To measure IRF, a certified colloidal LUDOX solution was used. IRF was taken into account when measuring the lifetimes of PL close to the duration of the laser pulse.

To calculate $\tau$ and to plot the time-resolved emission spectra (TRES), F900 software package (Edinburgh Instruments Ltd, UK) was used. TRES were obtained from the sequence of PL decays for different wavelengths of emission. This way, one can construct the PL spectra dependence on the delay time, $t_{d}$, following the maximum of the exciting laser pulse with picosecond temporal resolution. To plot TRES, kinetic PL decay curves were measured in the spectral range of 420-730 nm with increment of $2 \mathrm{~nm}$. The delay time for the constructed instantaneous PL spectra, $t_{d}$, was considered zero at maximum of the excitation laser pulse (IRF). 
The PL excitation-emission maps (PLE maps) obtained via combination of steady-state PL spectra at various excitation wavelengths were measured using a Horiba NanoLog excitation-emission spectrofluorometer equipped with a silicon detector.

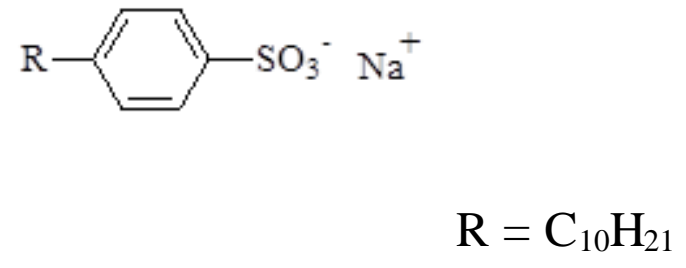

A

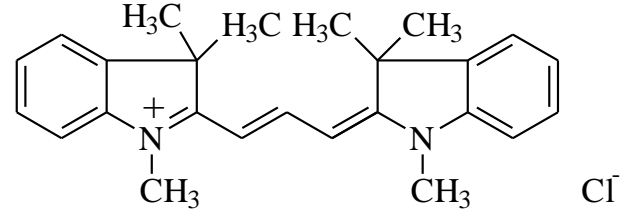

b

Fig. 1 Structural formulae of SDBS surfactant (a) and astraphloxin polymethine dye (b).

In our experiments, we used the following effective composite components influencing PL response of melanin. First, we selected sodium dodecylbenzenesulfonate (SDBS) surfactant as an anionic component of the composite (Fig. 1a) and astraphloxin belonging to the class of polymethine dyes as a cationic component (Fig. 1b). We studied aqueous solutions of natural melanin of plant origin obtained by an extraction [4-7]. The chemical structure of studied natural melanin is similar to that of synthetic melanin formed by 5,6-indolequinone monomers confirmed by chromatography and elemental analysis $[4,5]$. In addition, aqueous solutions of synthetic melanin (Sigma-Aldrich; \#M8631) were studied as a reference. The concentration of melanins in the solutions was $5 \cdot 10^{-5} \mathrm{wt} \%$. SDBS (Sigma-Aldrich; \#289957) was admixed to aqueous solutions of melanin at the premicellar concentration. The polymethine dye, astraphloxin, was obtained from the Institute of Organic Chemistry (NASU, Ukraine) and was added to the 
solutions of melanin at the concentration $5 \cdot 10^{-5} \mathrm{wt} \%$. The physical properties for aqueous solutions of SDBS and astraphloxin including absorbance and PL characteristics were studied before $[10,11]$. In this paper, we have studied aqueous solutions and mixtures for all the materials, and all our measurements were performed at room temperature $(\mathrm{T}=296 \mathrm{~K})$.

\section{RESULTS AND DISCUSSION}

The electronic properties of various melanin pigments have been studied spectroscopically [4-7], and in this paper we are focusing on elaboration of PL spectra and kinetics of water-soluble melanins and two-component systems of donor-acceptor complexes with charged molecules of SDBS surfactant or astraphloxin dye. Before study of two-component systems, we analysed the spectral characteristics of neat melanins of synthetic and natural origin. Fig. 2 shows the steady-state spectra of PL for solutions of natural (Fig. 2a, curve 1) and synthetic (Fig. 2a, curve 2 and Fig. 2b) melanins. Figure 3 shows the PLE maps plotting intensity of PL emission for a given excitation wavelength (vertical scale) and a given emission wavelength (horizontal scale) of aqueous solutions of natural (a) and synthetic (b) melanins. The color intensity scale is shown to the right of the graph. It can be seen from the PLE maps that the maximum PL intensity for both types of melanin falls in the emission range of $450-520 \mathrm{~nm}$, and the excitation maximum is localized in the spectral region of $380-420 \mathrm{~nm}$. As can be seen from Fig. 2 and 3, there are some common features in PL spectra of synthetic and 
natural melanins at different wavelength of excitation, $\lambda_{\mathrm{e}}$. Thus, we can see that PL spectra of both types of studied melanins are similar.

(a)

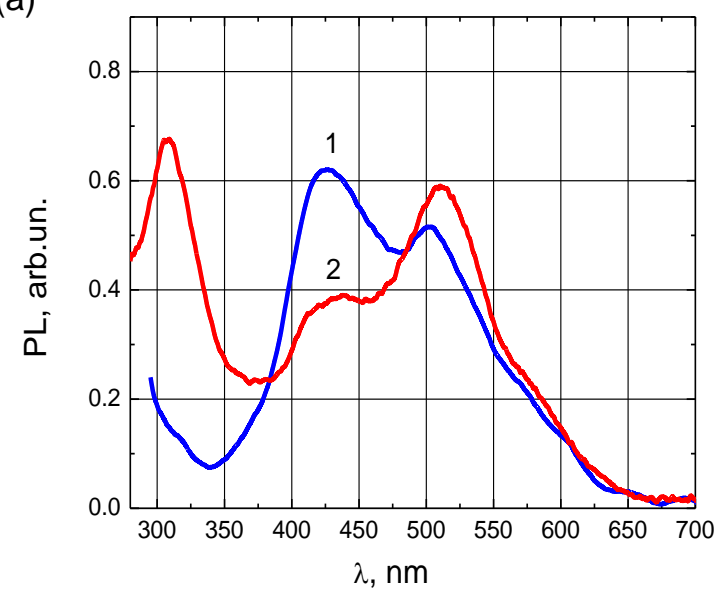

(b)

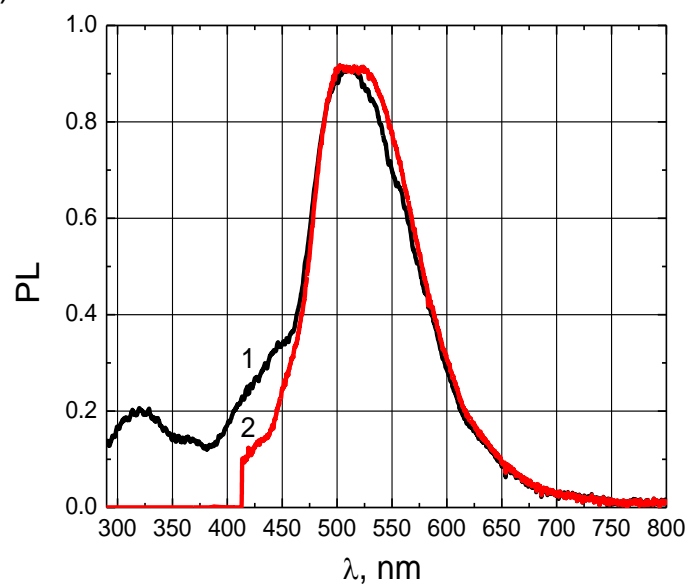

Fig. 2 (a) PL spectra for aqueous solutions of natural (1) and synthetic (2) melanins at $\lambda_{\mathrm{e}}=255 \mathrm{~nm}$ measured by LifeSpec II.

(b) PL spectra for aqueous solutions of synthetic melanin at $\lambda_{\mathrm{e}}=270 \mathrm{~nm}$ (1) and $385 \mathrm{~nm}$ (2) measured by USB 2000+UV-VIS-ES.
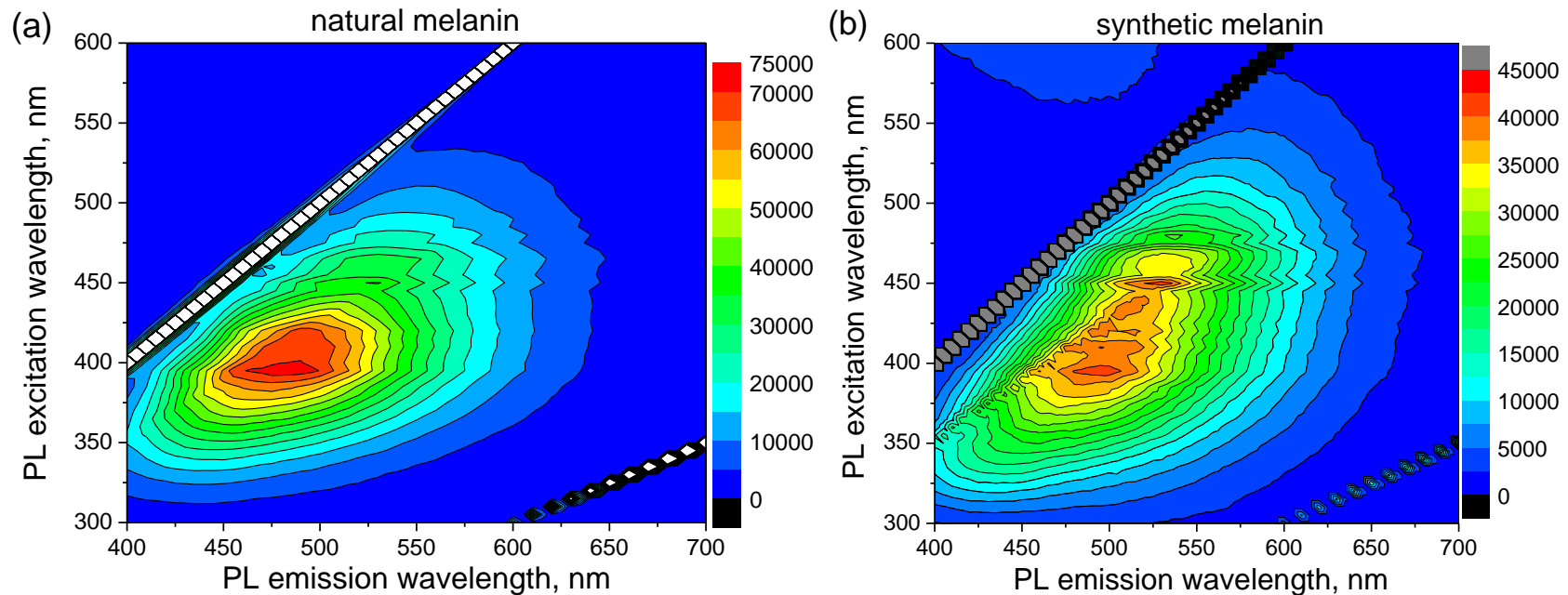

Fig. 3 PLE map for aqueous solutions of natural (a) and synthetic (b) melanins.

We have complemented the steady-state PL characteristics of investigated melanins with time-resolved PL study. Fig. 4 shows the PL kinetics of aqueous solutions of synthetic melanin excited at $\lambda_{\mathrm{e}}=255$ (Fig. 4a) and $405 \mathrm{~nm}$ (Fig. 4b). The dependencies of the PL intensity on the decay time $t_{d}$ were recorded over a 
wide range of emission wavelengths: $\lambda(\mathrm{PL})=315-680 \mathrm{~nm}$. Table 1 shows PL lifetimes, $\tau$, calculated from the measured kinetic curves. The contribution of each of the decay components to the PL spectrum is given as a percentage in adjacent columns.

(a)

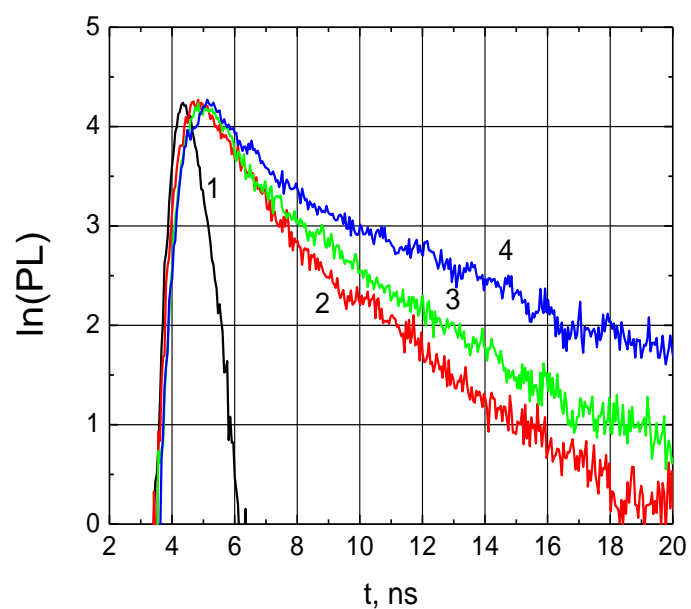

(b)

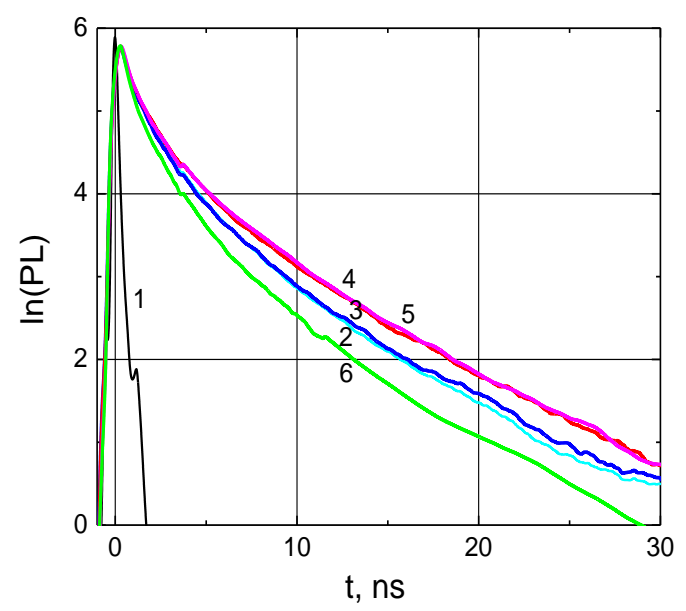

Fig. 4 (a) Kinetics of PL for aqueous solutions of synthetic melanin; $\lambda_{\mathrm{e}}=255 \mathrm{~nm}$; $\operatorname{IRF}(1) ; \lambda(\mathrm{PL})=315 \mathrm{~nm}(2), 440 \mathrm{~nm}(3)$, and $500 \mathrm{~nm}(4)$.

(b) Kinetics of PL for aqueous solutions of synthetic melanin; $\lambda_{\mathrm{e}}=405 \mathrm{~nm}$; IRF (curve 1). $\lambda(\mathrm{PL})=440$ (2), 460 (3), 500 (4), 520 (5), and 640 (6) nm.

Table 1. Lifetimes for PL of aqueous solutions of synthetic melanin for different wavelengths of PL emission, $\lambda(\mathrm{PL})$, and excitation, $\lambda_{\mathrm{e}}$.

\begin{tabular}{|c|c|c|c|c|c|c|}
\hline$\lambda(\mathrm{PL}), \mathrm{nm}$ & $\lambda_{\mathrm{e}}, \mathrm{nm}$ & $\tau_{1}, \mathrm{ps}$ & $\%$ & $\tau_{2}, \mathrm{ps}$ & $\%$ & $\chi^{2}$ \\
\hline 315 & 255 & 955 & 39 & 4140 & 61 & 1.078 \\
\hline 440 & 255 & 960 & 25 & 4210 & 75 & 1.177 \\
\hline 510 & 255 & 990 & 20 & 6580 & 80 & 1.046 \\
\hline 420 & 405 & 450 & 28 & 3300 & 72 & 1.908 \\
\hline 440 & 405 & 800 & 34 & 4780 & 66 & 1.634 \\
\hline 460 & 405 & 800 & 33 & 5890 & 67 & 2.155 \\
\hline 480 & 405 & 840 & 34 & 6100 & 66 & 2.118 \\
\hline 500 & 405 & 950 & 34 & 6730 & 66 & 2.285 \\
\hline 520 & 405 & 800 & 31 & 5460 & 69 & 2.073 \\
\hline 560 & 405 & 710 & 30 & 5690 & 70 & 1.497 \\
\hline 600 & 405 & 660 & 32 & 5270 & 68 & 1.404 \\
\hline 640 & 405 & 470 & 34 & 3890 & 66 & 1.730 \\
\hline 680 & 405 & 430 & 38 & 3200 & 62 & 1.458 \\
\hline
\end{tabular}


Our results show that the dynamics of PL decay for studied synthetic and natural melanins are similar in many respects. The PL decays have a two-component structure with fast and slow components of kinetic curves. The PL decays are well described by the two-exponential function

$$
\mathrm{I}_{\mathrm{PL}}(\mathrm{t})=\mathrm{A}_{1} \cdot \mathrm{e}^{-\mathrm{t} / \tau_{1}}+\mathrm{A}_{2} \cdot \mathrm{e}^{-\mathrm{t} / \tau_{2}}
$$

with PL lifetimes of $\tau_{1}$ and $\tau_{2}$ in the picosecond and nanosecond timescale, respectively. It should be underlined that the nanosecond PL component is predominant with contribution in the range of $60-70 \%$ (Table 1).

The investigated aqueous nano-suspensions of melanin are not stable and have tendency to aggregation. Surfactants can be used to prevent the aggregation process. Surfactants can accumulate at the liquid-solid interface of melanin macromolecules, and thus, reducing surface energy and limiting the aggregation into large particles. However the interaction of charged surfactant molecules with terminal reactive groups of the opposite sign promotes the formation of donoracceptor (D-A) complexes of specific melanin groups with surfactants and could lead to a change in the PL properties of melanins.

In our experiments we used anionic SDBS (Fig. 1a) as an effective surfactant for D-A complexation with melanin. An admixture of SDBS to an aqueous solution of melanin at the concentrations of surfactant below the critical micelle concentration (CMC) leads to an alteration in PL spectra of melanin (Fig. 5). For the initial colloidal aqueous solution of melanin, there are bands in the PL 
spectra with maxima at 336, 409, and $510 \mathrm{~nm}$ (Fig. 5, curve 1). A new intensive band at $390 \mathrm{~nm}$, which can be considered as a manifestation of the interaction between donor-acceptor macromolecules of melanin and SDBS is observed in PL spectra after admixture of the surfactant to the solution of melanin. The new band can be attributed to Coulomb interaction between the anions of SDBS and cations of melanin in the aqueous mixtures of SDBS and melanins (M). As a result of such interaction, intermolecular complexes of SDBS-M are formed.

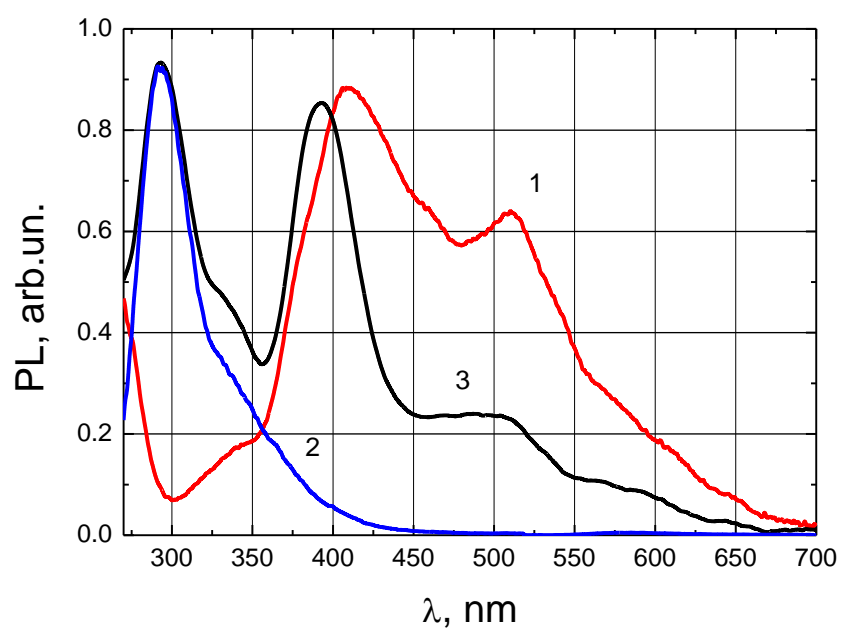

Fig. 5 Steady-state PL spectra of aqueous solutions of natural melanin (1), SDBS surfactant (2) and natural melanin with SDBS (3); $\lambda_{\mathrm{e}}=255 \mathrm{~nm}$; measured by LifeSpec II.

To understand the D-A complexation of M-SDBS, the aqueous solutions of SDBS were analyzed, which absorb light in the UV spectral region due to the presence of the benzene ring with easily excitable $\pi$-electrons. Absorption spectra of SDBS have a long-wavelength band at $260 \mathrm{~nm}$ corresponding to the lowest $\mathrm{HOMO} \rightarrow$ LUMO electronic transition associated with the benzene ring. In the PL spectrum 
of SDBS, bands at $292 \mathrm{~nm}$ and $340 \mathrm{~nm}$ can be distinguished (Fig. 5, curve 2). The PL spectral transitions are associated with benzene excitation and charge transfer between benzene and the charged SDBS fragment in the excited state. The band at $292 \mathrm{~nm}$ can be associated with the LUMO $\rightarrow$ HOMO benzene transition, and the $340 \mathrm{~nm}$ band is attributed to intramolecular charge transfer in the excited state of SDBS. The presence of a benzene ring in the SDBS molecule also contributes to its D-A adsorption on the melanin surface involving stack interaction and M-SDBS complexation.

To look at the temporal features of interaction between melanin and SDBS, we characterized the system via time-resolved PL. Kinetic curves of PL decays for aqueous solutions of melanin (M) and melanin with SDBS (M+SDBS) depending on $\lambda_{\mathrm{e}}$ and $\lambda(\mathrm{PL})$ are shown in Fig. 6. The PL decay curves for natural melanin and melanin with SDBS are slightly different in UV range of $\lambda_{\mathrm{e}}=255 \mathrm{~nm}$ and $\lambda(\mathrm{PL})=$ $400 \mathrm{~nm}$ (Fig. 6a) and are practically the same at longer $\lambda_{\mathrm{e}}$ and $\lambda(\mathrm{PL})$ (Fig. 6b). The kinetic curves are also well described by a two-exponential function with PL lifetimes in the picosecond and nanosecond ranges. The calculated lifetimes from the two-exponential dependences of PL for different $\lambda_{\mathrm{e}}$ and $\lambda(\mathrm{PL})$ are presented in Table 2. Comparison of the fast, $\tau_{1}$, and slow, $\tau_{2}$, components of the PL lifetime for solutions of various melanins and for melanin with SDBS (Table 1 and 2) shows slightly faster $\tau_{1}$ for synthetic melanins. In overall the changes of $\tau_{1}$ and $\tau_{2}$ at admixture of SDBS to melanin are not substantial with only $20 \%$ increase of $\tau_{2}$ observed at the PL excitation in the UV spectral range (Table $2 ; \lambda_{\mathrm{e}}=255 \mathrm{~nm}$, 
$\lambda(\mathrm{PL})=400 \mathrm{~nm})$. Thus, from two-exponent fitting of PL decays, the fast component of the PL lifetime for solutions of various melanins is changing in the range of 1000 ps, whereas the slow one varies from 3.2 unto 6.7 ns (Table 1 and 2).

(a)

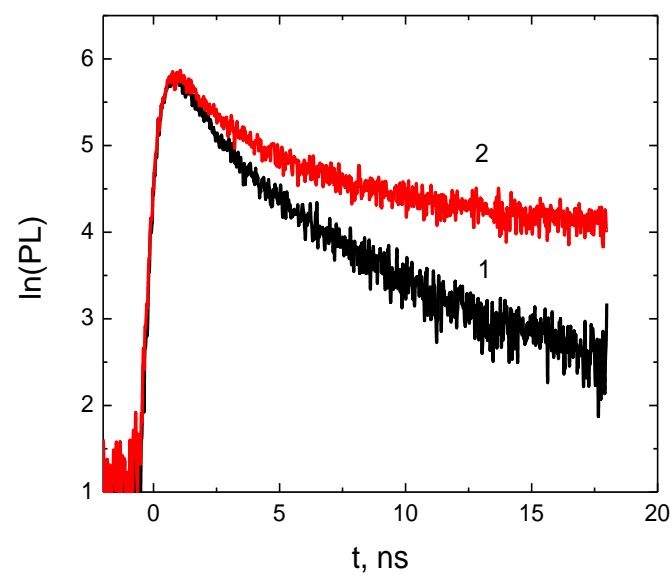

(b)

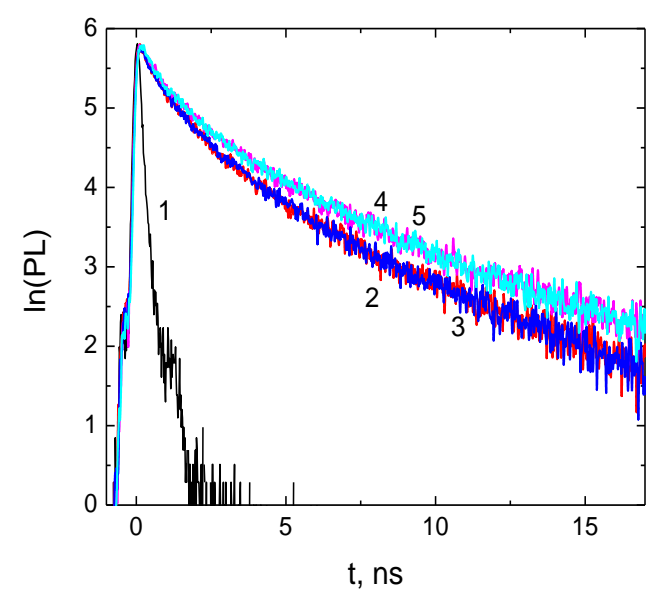

Fig. 6(a) PL kinetic curves for aqueous solutions of natural melanin (curve 1) and natural melanin with SDBS (curve 2). $\lambda_{\mathrm{e}}=255 \mathrm{~nm}, \lambda(\mathrm{PL})=400 \mathrm{~nm}$.

(b) PL kinetics for aqueous solutions of the natural melanin (curves 2,4) and melanin with SDBS (curves 3, 5); IRF - curve 1; $\lambda_{\mathrm{e}}=405 \mathrm{~nm}, \lambda(\mathrm{PL})=460(2,3)$ and $510(4,5) \mathrm{nm}$.

Table 2. Dependence of PL lifetime $(\tau)$ of natural melanin $(M)$ and natural melanin mixture with SDBS $(\mathrm{M}+\mathrm{SDBS})$ for different $\lambda_{\mathrm{e}}$ and $\lambda(\mathrm{PL})$.

\begin{tabular}{|c|c|c|c|c|c|c|c|}
\hline $\begin{array}{c}\lambda(\mathrm{PL}), \\
\mathrm{nm}\end{array}$ & $\lambda_{\mathrm{e}}, \mathrm{nm}$ & $\tau_{1}, \mathrm{ps}$ & $\%$ & $\tau_{2, \mathrm{ps}}$ & $\%$ & $\chi^{2}$ & \\
\hline 400 & 255 & 1380 & 25 & 5020 & 72 & 1.138 & $\mathrm{M}$ \\
\hline 400 & 255 & 1440 & 24 & 6140 & 76 & 1.050 & $\mathrm{M}+\mathrm{SDBS}$ \\
\hline 460 & 405 & 1064 & 28 & 4570 & 72 & 1.015 & $\mathrm{M}$ \\
\hline 460 & 405 & 1020 & 25 & 4360 & 75 & 1.177 & $\mathrm{M}+$ SDBS \\
\hline 510 & 405 & 1030 & 22 & 4840 & 78 & 1.042 & $\mathrm{M}$ \\
\hline 510 & 405 & 920 & 18 & 4640 & 82 & 0.970 & $\mathrm{M}+$ SDBS \\
\hline
\end{tabular}

Another focus of this article is to elucidate the mechanism of the melanin functionalization by polymethine dye aiming to tailor novel complexation material with enhanced electronic properties. Besides, such functionalization allows us to 
study interaction of melanins with various $\pi$-conjugated materials. We have used cyanine dye, astraphloxin, (Fig. 1b) for non-covalent functionalization of melanin. In solutions, the molecules of astraphloxin in the ground state, like many polymethine dyes, are trans-isomers [12]. The main element of the chromophore astraphloxin dye is an extended $\pi$-electron charge density alternating along the polymethine chain and alternatively redistributed during optical excitation [13]. The orders of $\pi$-bonds are aligned both in the ground state and in the excited state. PL of aqueous solutions of astraphloxin has a very low quantum yield and short lifetimes due to trans-cis conformational changes of the dye molecule following with effective nonradiative intermolecular processes. It is obvious that conformational changes in astraphloxin molecule also create steric hindrances to its aggregation. We did not observe the aggregation of astraphloxin molecules in aqueous solutions for concentrations upto $10^{-3} \mathrm{wt} \%$ [10]. The PL spectra of aqueous solutions of astraphloxin at small concentrations $\left(10^{-5}-10^{-6} \mathrm{wt} \%\right)$ have bands of 564 and $590 \mathrm{~nm}$ (Fig. 7a). At increasing concentration of astraphloxin, the intensity of the PL band at $564 \mathrm{~nm}$ slightly decreases, while the band of $590 \mathrm{~nm}$ increases and becomes broader. The PL lifetime of aqueous solutions of astraphloxin is practically independent of the emission wavelength $(\lambda(\mathrm{PL})=560-$ $610 \mathrm{~nm})$ and concentration $\left(\mathrm{C}=10^{-5}-10^{-3} \mathrm{wt} \%\right)$. At room temperature, the lifetime varies within $40-50$ ps (Fig. 8b, curve 1). Freezing of astraphloxin molecules or their adsorption on the surface increases the rigidity of the dye and creates steric hindrances for conformational changes in them. In this case, the 
measured PL lifetime is close to the radiative lifetime of the molecule. The radiative lifetime for frozen solutions of astraphloxin at $77 \mathrm{~K}$ is characterized by three components with $\tau_{1}=150-300 \mathrm{ps}, \tau_{2}=1.5-2.6$ ns and $\tau_{3}=3.5-10 \mathrm{~ns}$ [11].

(a)

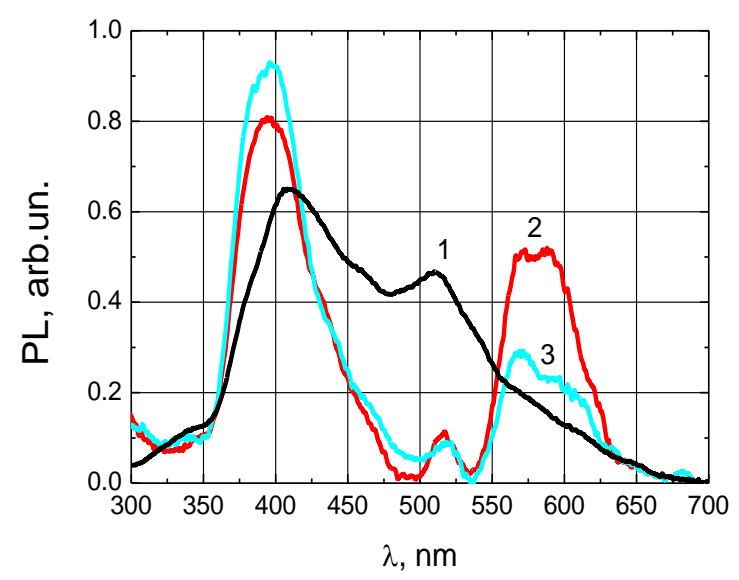

(b)

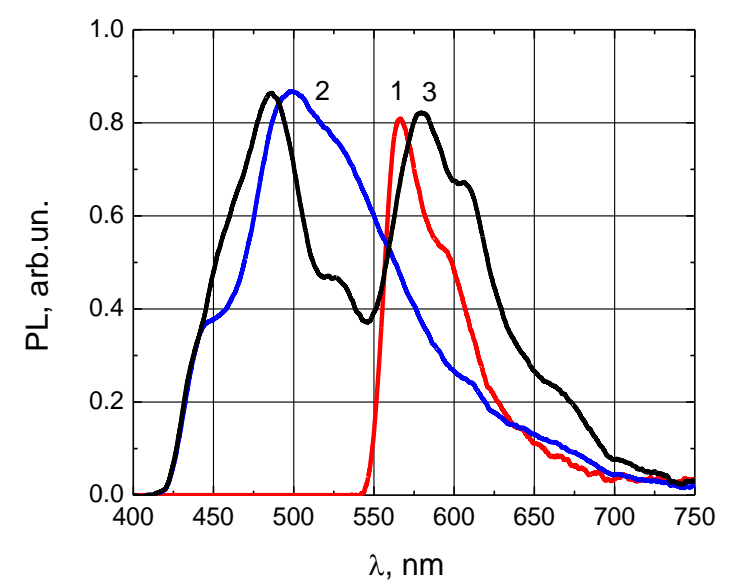

Fig. 7(a) Steady-state spectra of PL for aqueous solutions of natural melanin (1) and a mixture of natural melanin with astraphloxin (2-3) in water; $\lambda_{e}=255 \mathrm{~nm}$; measured by LifeSpec II.

(b) Steady-state spectra of PL for solutions of astraphloxin (1) and natural melanin (2) and a mixture of natural melanin and astraphloxin (3) in water; $\lambda_{\mathrm{e}}=$ $405 \mathrm{~nm}$; measured by USB2000+UV-VIS-ES.

Fig. 7a shows the steady-state PL spectra for solutions of natural melanin (1) and a mixture of melanin with astraphloxin (2-3) under UV excitation at $\lambda_{\mathrm{e}}=255$ $\mathrm{nm}$. In the PL spectra of neat melanin, we observe bands at 410 and $511 \mathrm{~nm}$, and addition of astraphloxin to natural melanin leads to significant changes in the PL spectrum. On the short wavelength side of the spectrum, an intense band at $394 \mathrm{~nm}$ appears, and in the long wavelength spectral region, we see the emission of astraphloxin. The astraphloxin emission has both molecular PL with band at 569 
$\mathrm{nm}$ and small aggregates PL with broad band in the range of 590-610 $\mathrm{nm}$ [10]. The new band at $394 \mathrm{~nm}$ emerged in the mixtures of the dye and melanin can be associated with the formation of D-A complexes between the molecules of astraphloxin and melanin nanoaggregates. Thus, in aqueous solutions of melanin with astraphloxin, an evidence of Coulomb interaction between the cations of astraphloxin and the anions of melanin is observed. The interaction leads to the formation of intermolecular complexes of astraphloxin-melanin.

To analyze the interaction of astraphloxin with melanin we have measured the PL spectra at different $\lambda_{\mathrm{e}}$. Figure $7 \mathrm{~b}$ shows the steady-state spectra of PL for solutions of astraphloxin (1), natural melanin (2), and mixtures of melanin with astraphloxin (3) at $\lambda_{\mathrm{e}}=405 \mathrm{~nm}$. In the PL spectra of the initial solution of melanin (Fig. 7b, curve 2), a band at $500 \mathrm{~nm}$ and a short wavelength shoulder at $450 \mathrm{~nm}$ can be discriminated. The initial solution of astraphloxin is characterized by the molecular spectrum of PL with a maximum at 566 and a shoulder at $590 \mathrm{~nm}$. Importantly, PL spectrum for the mixture of melanin with astraphloxin is not a superposition of spectra of two components. First, PL in the spectral range of melanin emission at $\lambda(\mathrm{PL})>500 \mathrm{~nm}$ is quenched; second, the emission featuring astraphloxin at $\lambda(\mathrm{PL})>550 \mathrm{~nm}$ also undergoes changes, where bands at 580, 608, and $660 \mathrm{~nm}$ appear. The new long wavelength bands can be associated with astraphloxin aggregation in the presence of melanin. Increasing of astraphloxin concentration in the mixtures with melanin leads to a significant aggregation of 
astraphloxin on the surface of melanin nanoclusters resulting in formation of naked-eye visible micro-particles and precipitation.

Measurements of PL kinetics for aqueous solutions of astraphloxin (Fig. 8b, curve 2), melanin (Fig. 8b, curves $3 \& 4$ ) and mixture of melanin with astraphloxin (Fig. 8a, curves 2-5) showed that the greatest changes of lifetimes occur in the range of astraphloxin emission. As in the case of mixtures of melanin with SDBS, the kinetic curves of PL for mixtures of melanin with astraphloxin in a broad spectral range of emission are well described by the two-exponential function with PL lifetimes in the picosecond and nanosecond timescales. The lifetimes $(\tau)$ of PL for astraphloxin (A), natural melanin (M) and melanin with astraphloxin $(\mathrm{M}+\mathrm{A})$ for different wavelengths of $\lambda(\mathrm{PL})$ are shown in Table 3. The lifetime of 40-50 ps for neat astraphloxin PL increases after its mixing with melanin up to $\tau_{2}=2330 \mathrm{ps}$ (Table 3, $\lambda(\mathrm{PL})=580 \mathrm{~nm})$. Such a change in the kinetics of astraphloxin radiation clearly indicates that the molecules of astraphloxin are adsorbed on the surface of melanin nanoclusters. The presence of a fast component with $\tau_{1}=90-120 \mathrm{ps}$ in the kinetics of astraphloxin radiation at $\lambda(\mathrm{PL})=580$ and $610 \mathrm{~nm}$ indicates that $\mathrm{J}$ like aggregates are formed on the surface of melanin nanoclusters. 
(a)

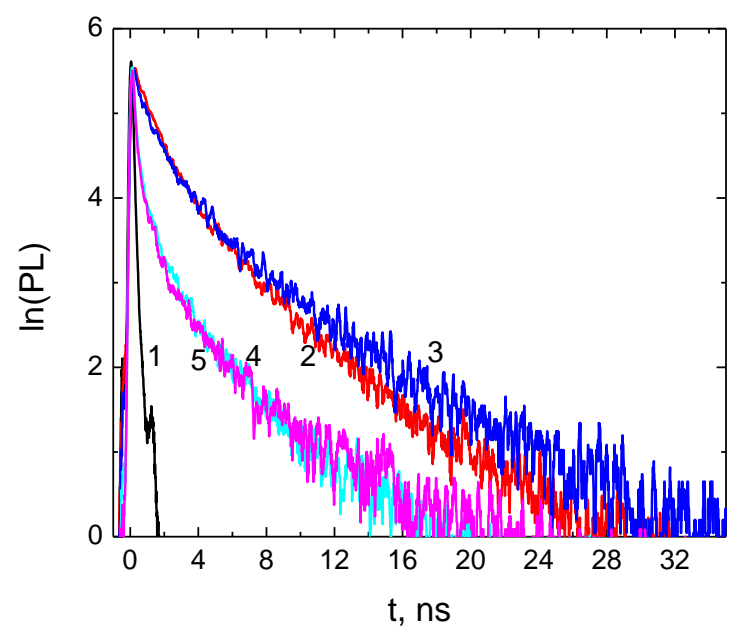

(b)

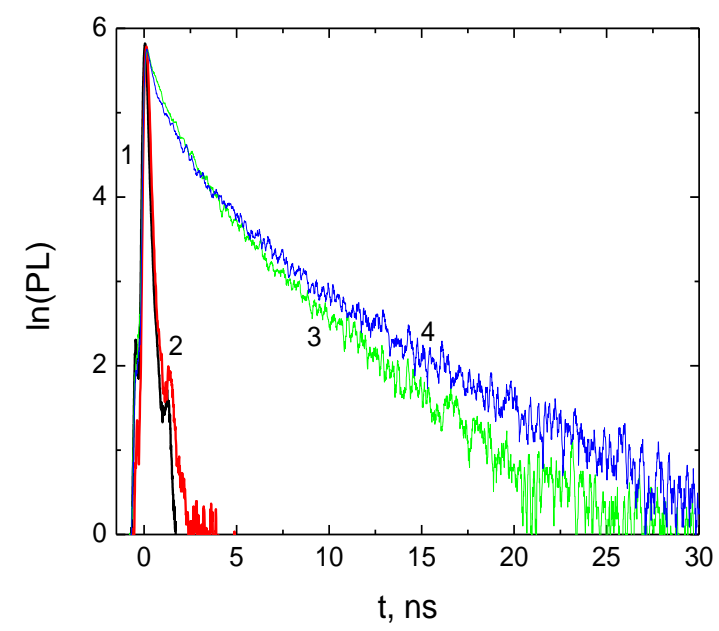

Fig. 8 (a) PL decays for aqueous mixture of natural melanin with astraphloxin at $\lambda(\mathrm{PL})=440$ (2), 520 (3), 580 (4), 610 (5); IRF (1).

(b) PL decays for aqueous solutions of both astraphloxin at $\lambda(\mathrm{PL})=570 \mathrm{~nm}$ (2) and natural melanin at $\lambda(\mathrm{PL})=440 \mathrm{~nm}(3), 520 \mathrm{~nm}$ (4); IRF (1). $\lambda_{\mathrm{e}}=405 \mathrm{~nm}$.

Table 3. Dependence of the lifetime $(\tau)$ of PL of astraphloxin (A), natural melanin $(\mathrm{M})$ and mixture of melanin with astraphloxin $(\mathrm{M}+\mathrm{A})$ for different $\lambda(\mathrm{PL})$.

\begin{tabular}{|c|c|c|c|c|c|c|c|}
\hline$\lambda(\mathrm{PL}), \mathrm{nm}$ & $\lambda_{\mathrm{e}}, \mathrm{nm}$ & $\tau_{1}, \mathrm{ps}$ & $\%$ & $\tau_{2,} \mathrm{ps}$ & $\%$ & $\chi^{2}$ & \\
\hline 570 & 405 & 40 & 100 & - & - & 1.120 & $\mathrm{~A}$ \\
\hline 440 & 405 & 580 & 24 & 4650 & 76 & 1.282 & $\mathrm{M}$ \\
\hline 510 & 405 & 640 & 25 & 4760 & 75 & 1.245 & $\mathrm{M}$ \\
\hline 440 & 405 & 920 & 30 & 4270 & 70 & 1.197 & $\mathrm{M}+\mathrm{A}$ \\
\hline 520 & 405 & 630 & 21 & 4220 & 79 & 1.111 & $\mathrm{M}+\mathrm{A}$ \\
\hline 580 & 405 & 120 & 40 & 2330 & 60 & 1.164 & $\mathrm{M}+\mathrm{A}$ \\
\hline 610 & 405 & 90 & 41 & 2280 & 59 & 1.194 & $\mathrm{M}+\mathrm{A}$ \\
\hline
\end{tabular}

Fig. 9 shows TRES spectra at $\lambda_{\mathrm{e}}=405 \mathrm{~nm}$ for mixtures of melanin with astraphloxin at different delay times, $t_{d}$. The spectra were normalized on the PL band of melanin having the longest lifetime with maximum at $\lambda(\mathrm{PL})=470 \mathrm{~nm}$ and a shoulder at $525 \mathrm{~nm}$. It is seen from the presented spectra that the shape of PL contribution of melanin interacting with astraphloxin practically does not change in the range of $t_{d}=0-3000 p s$. The instant spectral components of astraphloxin 
peaking at $\lambda(\mathrm{PL})=590-595 \mathrm{~nm}$ correspond to the aggregated forms of astraphloxin on the surface of melanin nanoclusters. Importantly, the instantaneous spectra of PL in the region of astraphloxin radiation depend strongly on $t_{d}$. At $t_{d}=0$ $-500 \mathrm{ps}$, the PL emission of astraphloxin is predominant in the PL spectra (Fig. 9a, curves 1-4). At $t_{d}>800 \mathrm{ps,} \mathrm{the} \mathrm{instantaneous} \mathrm{PL} \mathrm{spectra} \mathrm{are} \mathrm{dominated} \mathrm{by} \mathrm{the}$ emission of melanin nanoclusters. In the Fig. 9 combining PL spectra and differential spectral dependences, it is possible to distinguish emission the shoulders at $570 \mathrm{~nm}$ corresponding to molecular emission of astraphloxin and the bands with maxima at $590-595 \mathrm{~nm}$ followed with long wavelength shoulders in the range of $600-700 \mathrm{~nm}$, which correspond to the emission of aggregated form of astraphloxin.

(a)

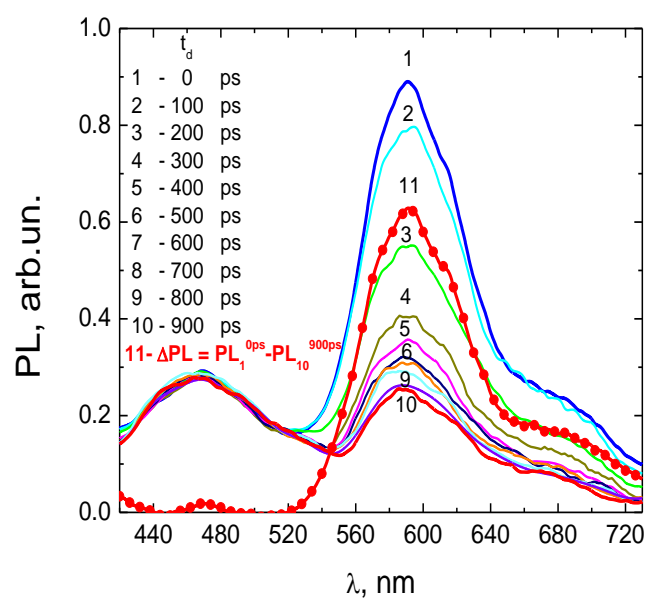

(b)

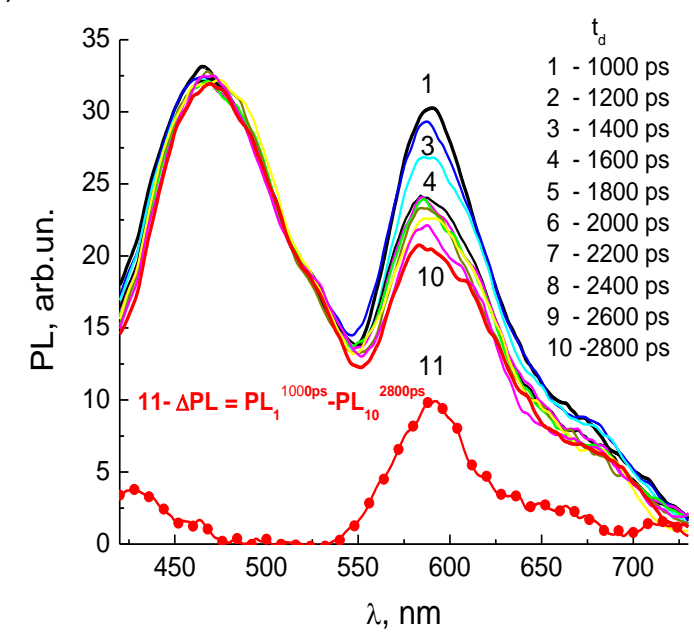

Fig. 9 Time-resolved emission spectra (TRES) of aqueous mixtures of natural melanin with astraphloxin with delay times, $t_{d}$, ranging (a) from 0 to $900 \mathrm{ps}$ with increment of $100 \mathrm{ps}$ and (b) from 1000 to 2800 ps with increment of $200 \mathrm{ps}$. 
As noted above, on one hand, astraphloxin interacts with macromolecules of melanin forming D-A complexes, and, on the other hand, molecules of the dye are adsorbed on the surface of melanin forming various types of nanoaggregates. Such absorption of astraphloxin on the surface of melanin macromolecules can be used in fluorescence microscopy to label specific areas of study. Besides, interaction of melanin with astraphloxin helps us to elucidate the nature of electronic transitions in melanin nanostructures featuring properties of organic crystals with charge transfer (CT crystals). The excited states in organic CT crystals are well described by wave functions delocalized within two molecular disks in the axial direction of the stack $[14,15]$. Such delocalization of excitation occurs due to the large value of the overlap integral between $\pi$-orbitals of adjacent disks and small distance between the disks. The delocalization of excitation distinguishes CT crystals from other molecular crystals in which the electron-hole pairs are localized on one molecule. Besides, this phenomenon places $\mathrm{CT}$ crystals in an intermediate class between ordinary molecular crystals and semiconductor crystals. In CT crystals, the energy-level scheme has CT transitions shifted towards lower energies and thus located lower than the Frenkel molecular exciton states (Fig. 10) [14]. 


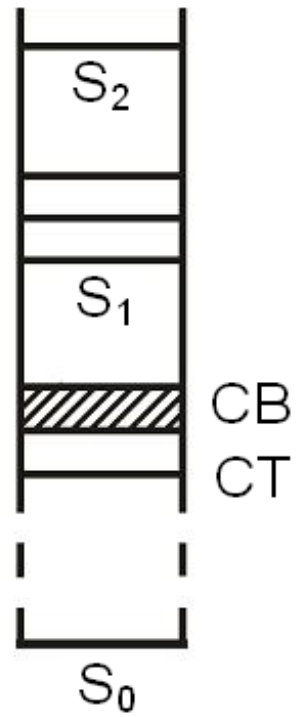

Fig. 10. Electronic diagram of melanin.

CT denotes charge-transfer level and CB stands for conduction band.

One more difference from typical molecular crystals can be distinguished for melanin nanoclusters. Within the macromolecular disk, the absorption of light by conjugated sections of copolymers of different lengths plays important role. In this respect, excited states in molecular disks are similar to excitations in conjugated polymers. At the initial stage of excitation in conjugated polymers, Frenkel excitons are formed, which can then be transformed into CT states. As in conjugated polymers, the elementary region of localization of a Frenkel exciton is the $\pi$-conjugate section of the macromolecule [16]. If Frenkel exciton in conjugated polymers is transformed into the $\mathrm{CT}$ state between two adjacent conjugated polymers, then the macromolecular Frenkel state in the melanin nanoclusters is transformed into the CT state between two macromolecular disks. It can be assumed that, as in CT crystals, both free and localized CT excitons can occur in melanin nanoclusters. Such states can manifest themselves in both 
photoconductivity and PL spectra. Taking into account a small strength of the transition oscillator for CT transitions, CT states will be characterized by long lifetimes in the PL spectra. Furthermore, considering a periodic structure of nanoclusters with a distance between molecular disks of $0.35 \mathrm{~nm}$, the CT-states with radius of $0.35 \mathrm{~nm}$ should make a significant contribution to PL.

One of the confirmations of the presented model for melanin nanoclusters excitation is that the PL kinetic curves of melanin, melanin with SDBS or astraphloxin in a wide spectral range of radiation are well described by a twoexponential function with PL lifetimes in the picosecond and nanosecond timescales. In optical excitation of melanin nanoclusters, molecular Frenkel excitons appear at the initial stage of excitation in molecular disks. Some of these excitons transit into the radiative state. The other part of molecular excitons is nonradiatively transformed into free CT excitons. Free CT excitons, interacting with structural defects, are transformed into localized CT excitons. The picosecond range of PL lifetimes is characteristic for radiative lifetime of free excitons, whereas the nanosecond timescale of lifetimes are featured in localized CT excitons. As can be seen from PLE map (Fig. 3), a well-defined spectral region of 360-420 nm can be attributed to states through which molecular excitons can form. Covering melanin nanoclusters with SDBS or astraphloxin molecules, which are effective traps for surface excitons, one can distinguish the emission of CT excitons from the nanocluster bulk area. Thus, the broad band in the emission range of $450-520 \mathrm{~nm}$ in the PL spectra of melanin-astraphloxin mixtures (Fig. 9) 
can be attributed to the emission of $\mathrm{CT}$ excitons from the bulk of the melanin nanoclusters.

For PL spectra of aqueous solutions of melanin with astraphloxin at $\lambda_{\mathrm{e}}=405$ $\mathrm{nm}$ in short-wavelength spectral region with respect to the emission of astraphloxin, changes are observed that can be related to the formation of intermolecular melanin complexes and astraphloxin. In this spectral region (500$520 \mathrm{~nm}) \mathrm{PL}$ of the melanin is quenching and a new intense band at $490 \mathrm{~nm}$ appears. Such changes in PL spectra of aqueous solutions of melanin were observed experimentally at low-temperature $(77 \mathrm{~K})$ spectral studies [4-7]. These changes were explained by the presence of pre-dimer states in melanin nanoclusters, which are acting as the traps of free excitons and being followed by relaxation to excimer emission $(\lambda(\mathrm{PL})>500 \mathrm{~nm})$. It is obvious that in mixed aqueous solutions of melanin with astraphloxin, the molecules of astraphloxin effectively interact with pre-dimeric states on the surface of melanin nanoclusters and form intermolecular complexes with charge transfer. This leads to the quenching of excimer emission and an increase in the intensity of the emission of free excitons.

\section{CONCLUSIONS}

Steady-state and time-resolved PL spectra of natural and synthetic melanin, as well as melanin composites with SDBS surfactant and astraphloxin polymethine dye have been studied. We showed that PL spectra and PL decays for synthetic and 
natural melanins are similar in many aspects. The kinetic curves of PL are well described by the two-exponential function with PL lifetimes in the picosecond and nanosecond ranges. Thus, the dynamics of PL decay have a two-component structure with fast and slow components.

Our spectral and kinetic studies of PL for neat melanin and mixtures of melanin with anionic (SDBS) and cationic (astraphloxin) molecules showed that intermolecular charge transfer complexes and molecular mixed-type nanoaggregates are formed. Molecules of astraphloxin, having an extended $\pi$ electron system, form J-like aggregates on the surface of melanin nanoclusters. This is confirmed by the changes in both the spectra and kinetics of PL for mixture of astraphloxin with melanin. Particularly, the PL lifetime for neat astraphloxin is $40-50$ ps regardless of the concentration. In mixtures of melanin with astraphloxin, we observed PL of astraphloxin nanoaggregates in the spectral region of 580-680 nm featured with two lifetimes at about $\tau_{1}=90-120 \mathrm{ps}$ and $\tau_{2}=2.2-$ 2.4 ns.

The formation of intermolecular complexes with charge transfer of melanin with anionic (SDBS) and cationic (astraphloxin) molecules in mixed aqueous solutions leads to significant changes in the PL spectra of mixed solutions. At UV range excitation of aqueous solutions of melanin with anionic SDBS, at the concentration below CMC, a $394 \mathrm{~nm}$ band appears in the PL spectra, which can be associated with the formation of intermolecular CT-complexes. Besides, there are clear evidences of formation of intermolecular complexes between melanin and 
astraphloxin, where the dye molecules effectively interact with pre-dimeric states on the surface of melanin nanoclusters and form intermolecular CT-complexes. As result of such interaction, we observe quenching of excimer emission and an increase in the intensity of the emission of free excitons in melanin nanoclusters.

Acknowledgements.

P.M.L. and A.G.R. acknowledge support of Royal Academy of Engineering / The Leverhulme Trust (Senior Research Fellowship, \#LTSRF1617/13/57).

\section{REFERENCES}

1. McGinnes, J. (1972). Science, 177, 896.

2. Rosei, M., Moska, L., Galuzzi, F. (1996). Synth. Met., 76, 331.

3. McGinnes, J., Corry, P., Proctor, P. (1974). Science, 183, 853.

4. Davidenko, S.A., Kurik, M.V., Piryatinskii, Yu.P., Verbitsky, A.B. (2005). Mol. Cryst. Liq. Cryst., 426, 37.

5. Grishchuk, V.P., Davidenko, S.A., Zholner, I.D., Verbitskii, A.B., Kurik, M.V., Piryatinskii, Yu.P. (2002). Tech. Phys. Lett., 28, 896.

6. Davidenko, S.A., Kurik, M.V., Piryatinskii, Yu.P., Verbitsky, A.B. (2008). Mol. Cryst. Liq. Cryst., 496, 82.

7. Verbitsky, A.B., Rozhin, A.G., Lutsyk, P.M., Piryatinski, Yu.P., Perminov, R.J. (2014). Mol. Cryst. Liq. Cryst., 589, 209.

8. Larsson, B.S. (1993). Pigment Cell Res., 6, 127. 
9. Apanasovich, V.V., Novikov E.G. (1992) J. Appl. Spectroscopy. 56, 538.

10. Lutsyk, P., Piryatinski Yu., AlAraimi M., Arif R., Shandura M., Kachkovsky O., Verbitsky A., Rozhin A. (2016) J. Phys. Chem. C., 120, 20378.

11. Lutsyk, P., Piryatinski, Yu., Kachkovsky, O., Verbitsky, A., Rozhin, A. (2017). J. Phys. Chem. A., 121, 8236.

12. Chibisov, A.K., Zakharova, G.V., Gerner, H. et al. (1995). J. Phys. Chem., 99, 886.

13. Kachkovsky, A.D. (1997). Uspekhi Khimii, 66, 715 (In Russian); Kachkovskii, A.D. (1997). Russ. Chem. Rev., 66 (8), 647 (In English).

14. Agranovich, V.M., Hochstrasser, R.M. Eds. (1983). Spectroscopy and excitation dynamics of condensed molecular systems, Elsevier Science Pub.Co, North-Holland Pub. Co.: Amsterdam, New York, USA, Netherlands.

15. Knoester, J. and Agranovich, V.M. (2003) Electronic Excitations in Organic Based Nanostructures. In: Agranovich, V.M. and Bassani, G.F., Eds., Thin Films and Nanostructures, Elsevier, Amsterdam, Vol. 31.

16. Chang, R., Hsu, J.H., Fann, W.S., et al. (2000). Chem. Phys. Lett. 317, 142. 